\title{
Research and Design of CAE Technology Service Platform for Small Mechanical Manufacturing Enterprises
}

\author{
Lingli Zhu" a and Guofang Kuang ${ }^{1}$ \\ ${ }^{1}$ College of Information Technology, Luoyang Normal University, Luoyang, 471934, China \\ azhulinglils@163.com
}

Keywords: CAE; Public technical service; Machinery manufacturing; Expert system; Service platform

\begin{abstract}
In view of the small machinery manufacturing enterprise's characteristic and the actual demand in CAE by study of CAE secondary development language APDL, the effective integration of $\mathrm{CAD}$ and $\mathrm{CAE}$, and the expert system is introduced to the results of CAE analysis processing. The CAE public service platform for small enterprises, which is the main content of the previous treatment, post processing, and the result of intelligent processing, is constructed. The platform to achieve the function of intelligent analysis and processing results, no need to guide the background of the engineer, the CAE public service technology to a new level.
\end{abstract}

\section{Introduction}

For small machinery manufacturing enterprises, CAE software such as ANSYS, Adams and NASTRAN and so on, is not the core business, but the use of CAE software to improve the quality of products, shorten the product design cycle, plays a very important role in improving the overall competitiveness of enterprises [1]. Considering the common needs of CAE software itself characteristics and small and medium-sized enterprises in terms of information exists, it is not difficult to see in ASP platform as a medium, between colleges and universities and small businesses establish sharing CAE software of bridge is very necessary. At the same time, with the rapid development of network technology, network bandwidth is increasing, so that the application of remote CAE provides hardware support.

The establishment of based on web remote CAE service target is to make full use of the existing network technology, computer technology and modern information technology, to establish a small business CAE service, web applications, users can remote access to the system, thus providing a model is convenient, economical and efficient CAE service mode. Therefore, it is of great significance to provide information and technical support for the general small manufacturing enterprises through the Web service model, and to improve the overall competitiveness of small manufacturing enterprises and the upgrading of the industrial information.

\section{Analysis and Design of Service Platform System Function}

Pre Processing Module. The key parameters for the unfamiliar command line of the primary user of the case, the user only needs to submit the model, modeling and analysis of process have the server, to improve the efficiency of modeling, and reduces the requirements on the user's level of expertise. The system adopts the model of parametric analysis and the model of friendly operation interface. Input from the user model file, to the grid division, and constraints and the applied load, friendly graphical interface to guide the user, users only need to enter or select the parameters needed for analysis, don't need to understand the command file generation process, the system automatically generated can be performed by the command file [2].

System with complete function package, the user in the environment of ANSYS to use all functions of packaging for the macro, in the client in accordance with the design steps to fill in the parameters, system will automatically related macro assembly for the command file, the system will background to 
generate the command file is submitted to the server, the server invokes the corresponding macro complete finite element analysis.

This model is the calculation of the conventional and calculation needed for a short time, establishment of the model is easy, the user opens the analysis service system of home manufacture, according to the web page provided by the calculation of the hyperlink, choose to calculate the, according to the requirement of input parameters, in the browser will computational results are obtained.

Post Processing Module. The results of finite element analysis processing are to view the results calculated by CAE software. In a sense, it may be the most important part of the whole analysis process [3]. After the user through the processing of this tool to analyze the results of the calculation, you can determine the structure of the state of behavior.

The finite element calculation results are a group of huge data, to get useful results from this group of data, the first to have to deal with the data. Data itself is meaningless; it is only a symbol, only for the purpose of the interpretation of the symbol (that is, the processing) can give the symbol to the specific meaning [4]. The remote CAE post processing system based on ASP receives the results from the finite element analysis, and then processes the results.

Results Intelligent Processing Module. The results of CAE analysis, which is easy to understand and can be easily understood, have great reference value to the users. But for the primary user, the results of the CAE analysis can only rely on experience to optimize the data, and the experience of only the objective and not accurate. So it is necessary to optimize the CAE analysis results [5]. The expert system technology into field of CAE technology, using the ability of thinking and reasoning, accomplish a great deal of information analysis and processing on behalf of the user, directly provide a guiding significance for the process of conclusions and recommendations, will effectively solve the complexity and the users of the CAE system using the contradiction between demands for simplicity, shorten the distance between the user and the CAE system. According to the above analysis, the remote CAE post-processing system is divided into the following main modules: pre-processing module, finite element analysis module, post-processing module, and the results of intelligent processing module. As shown in Fig. 1.

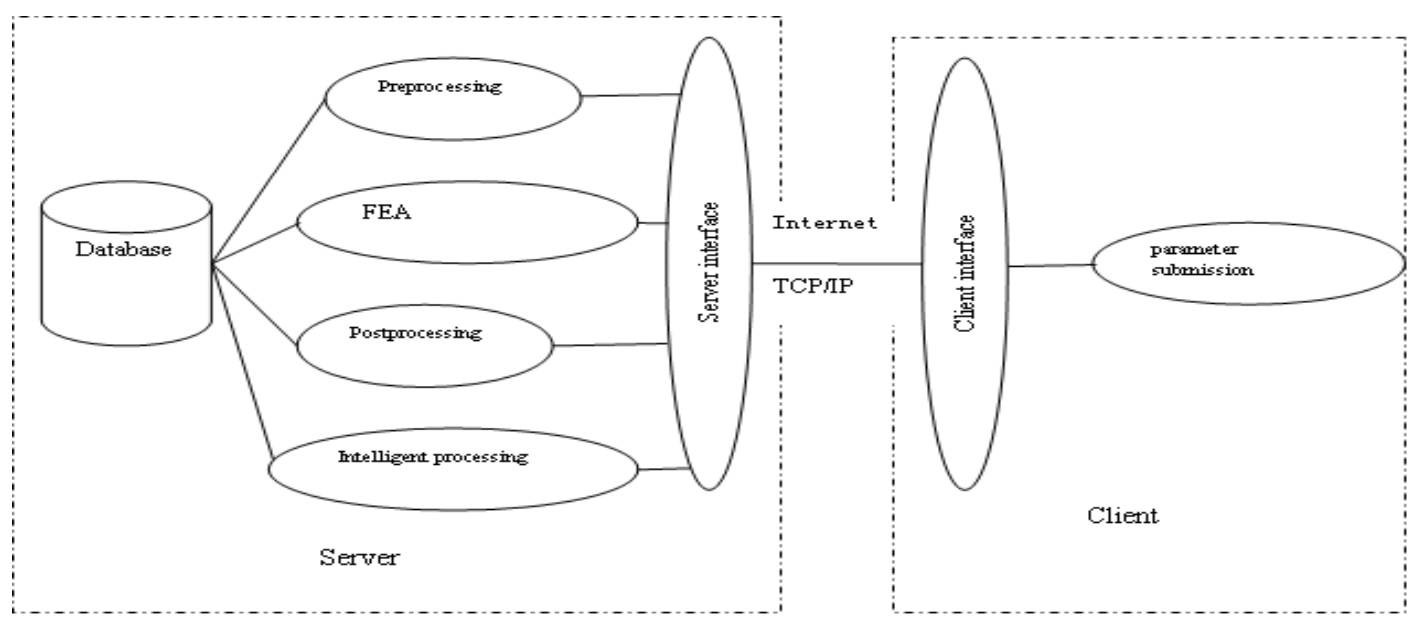

Figure 1. Remote CAE post processing system module chart

\section{Research on Key Technologies of Service Platform}

Using APDL Language for the Two Development of ANSYS. The two development features of CAE include four components: the parametric design language (APDL), the user interface design language (UIDL), the user program characteristics (UPFS), and the ANSYS data interface [6].

ANSYS parameter design language APDL (ANSYS parametric design language), with macros, circulation, the branch such as programming language function, and can provide a simple interface 
customization features, can achieve interactive parameter input and run the program, which can realize the parameterized modeling.

APDL is an explanatory language, can make ANSYS through the variable design language to develop, in order to give full play to the potential of ANSYS. APDL is a kind of command language, which is very similar to the FORTRAN language, it can use it to perform the tasks of general purpose, and even set up their own model according to the order. APDL also includes many other features, such as repeating a certain command, macro, then-else if branch, do loop, scalar, vector, matrix operations, etc.. The use of APDL can be more effective in the analysis and calculation, it can achieve the user's work (cycle, branch, macro and other structures) automation, is an efficient means of parametric modeling [7]. APDL is not only the classic characteristics of design optimization and adaptive mesh generation, but also provides a lot of convenience for daily analysis.

According to the requirement of the mechanical parts of the structural design features and analysis, the parameters for describing the feature size, and in establishing the finite element model and analysis, parameters that characterize the process, in order to achieve variable structure parameters of finite element analysis. This is essentially a parametric design using APDL language description method for structural design, and then carries on the finite element analysis method.

The scheme of this paper is to describe the finite element model of mechanical components. CAE simulation system automatically generates the finite element model language; abstract the feature parameters instead of the parameters in the description language, so as to realize the finite element dynamic modeling of variable structure parameters.

CAE simulation system of mechanical components with APDL language is to establish the entity modeling, analysis process, the results of the process of finite element modeling and analysis process flow [8]. APDL is used to replace the parameters in the model, which is abstracted by the characteristic parameter, which is the modeling process of the variable parameter.

CAD/CAE Integration Framework. In the project, a complete part design process generally includes the physical structure design and the strength, the rigidity, the deformation and so on various kinds of analysis. Is a traditional method, design personnel according to the requirements of the functional parts, first in the mind formation concept model, and then express in the form of two-dimensional engineering drawings, and it carries on the analysis according to the experience and the related standard.

Based on the current level of technology development, CAD/CAE integration has not yet reached the stage of intelligence, the need for technical personnel to set up and intervene in order to ensure that products in the design stage can achieve optimization. Fig. 2 expresses the overall process of $\mathrm{CAD} / \mathrm{CAE}$ integrated design.

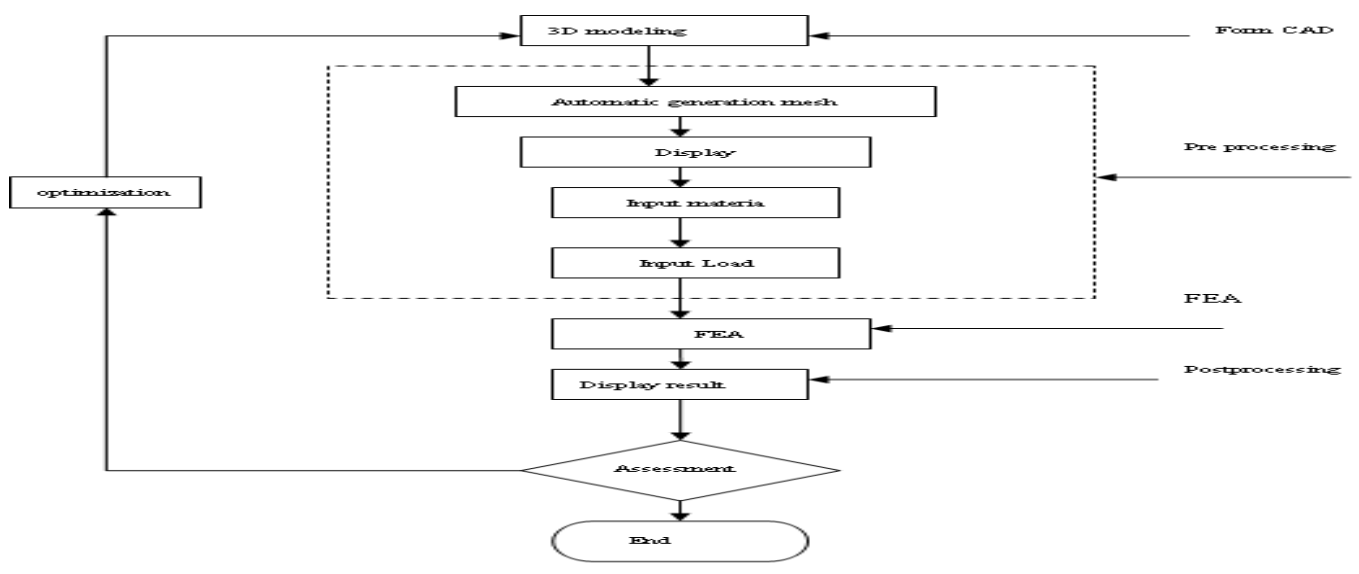

Figure 2. CAD/CAE integration flow chart

In the CAE input CAD system to establish the model, mainly through the following two ways: (1) in the CAD system to establish the model, they are stored as IGES format files, and then enter the CAE 
system. (2) The model of system is directly delivered to the CAE system through the parameterization of the interface [9]. Although this method for service platform developers, and it is the workload is large, but for the primary user, the more intuitive and more intuitive. So the platform chooses second ways.

Construction of Knowledge Base. Establishing the knowledge base is data intelligent processing module of the important link, the design of knowledge base requirements can effectively and accurately express the special field knowledge, with modular structure, and corresponding reasoning confidential cutting, to facilitate the interpretation of knowledge, easy to manage and maintain.

The field in the rule table is mainly composed of the rule name, the rule condition, the conclusion and so on. In this system, it is expressed in three tables. The rules of the table and the consequent rule connection are table [10]. All parts of the rules referred to in the preceding paragraph table if end, composed of three field ifID (rules referred to in the preceding paragraph shall be the serial number), paraID (antecedent parameters related to the serial number), condition (rule conditions). Consequent table to then end, composed of thenID (consequent serial number), paraID (rule consequent parameters of the serial number), ifNum (the number of rule conditions) and conclusion (rule conclusion) field. Rule connection table to rule ending, composed of ruleID (rule sequence number), ifID (antecedent sequence number), thenID (consequent sequence number).

The dynamic database is also called the global database, which is used to store the information of the original feature data in the problem domain, and to get all kinds of intermediate information in the process of reasoning and solve the problem. Dynamic database is dynamic and changeable, and its content is constantly changing. Reasoning machine according to the contents of the database from the knowledge is base to select the appropriate knowledge of reasoning, and then the results of the introduction of the database into the database. In ASP based remote CAE post-processing system, the parts of the dynamic data table is process at the end of the table, only contains a midresult field. In each reasoning process, first remove the contents stored in the midresult field in the table, and then store the intermediate results or the final results of the reasoning.

\section{System Implementation}

In the process of system development, the programming language used is Visual C. Visual C is a part of visual studio. Net compiler platform, is one of the key development tools of the. Net platform. It provides a complete interface to access the Microsoft. Net platform. Visual $\mathrm{C}$ is a modern and innovative programming language and tools can create connected to the.net software for Microsoft Windows, Internet and various kinds of equipment.

The system can be divided into the pre processing interface, the post processing interface and the intelligent result processing interface. The user can easily operate the model according to the actual input parameters, as is shown by Fig. 3.

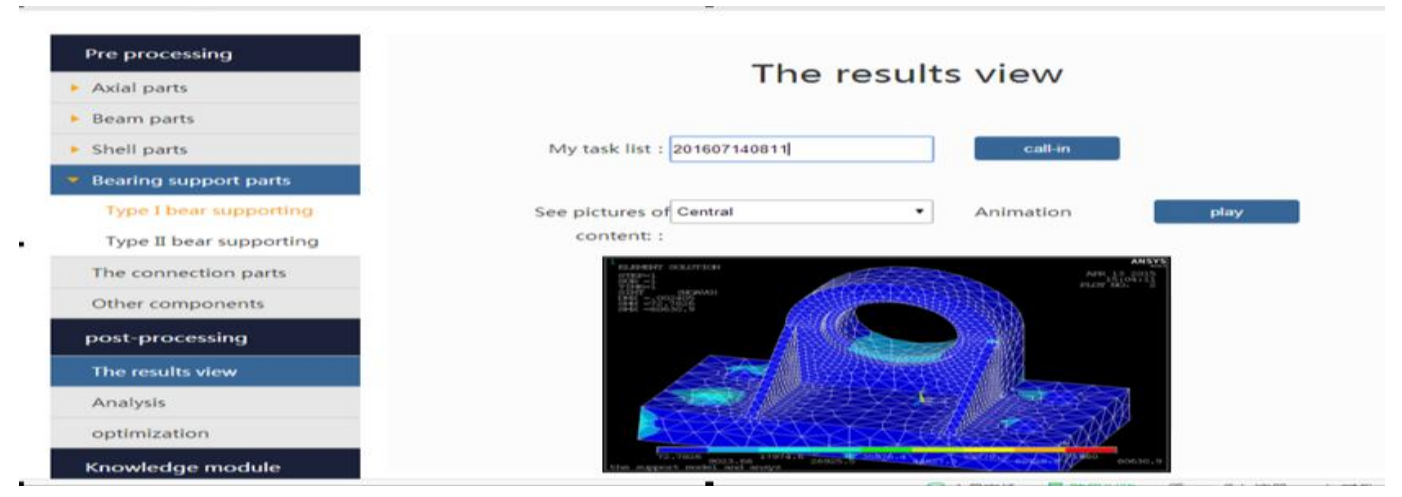

Figure 3. Post processing interface 


\section{Summary}

Through the study of integrated CAD / CAE technology, the CAE secondary development technology and expert system was developed for small and medium-sized enterprise CAE Public technology service platform, the platform based on network, suitable for remote users using CAE application. Enterprises can flexibly the CAE application server placed in a certain position, as long as you can connect to the Internet, any of the remote user can for remote analysis and view the results, don't need backend engineer online support, improve the running efficiency.

\section{Acknowledgement}

This paper is supported by Science and technology project of Henan Province (142102210475, 152102210123).

\section{References}

[1] Robert George. Gehling, Development of A General Application Service Provider (ASP), Doctor of Philosophy May 11, 2002.

[2] Jae Geun Ahn, Choon Seong Leem, Jung Hwan Yang. A Framework for Certification and Audit of Application Service Provider-ASP Springer Netherlands Volume 10, Number 3,239-252.

[3] S. J. Shepard, "IT shops takes stock of application service providers", IT Professional 2(2), pp. 12-16, 2000.

[4] Chris Bennett, Greg T Timbrell. Application Service Providers: Will They Succeed. Springer Netherlands Volume 2, Number 2 195-211, August 2000.

[5] Masahiro Nakata, Katsuyuki Yasuda. Quality Assurance Activities for ASP Based on SLM in Hitachi Springer Berlin / Heidelberg: Lecture Notes in Computer Science Volume 2349/2002 82.

[6] P. Bacquet, O. Brun, J.M. Garcia, et al. Telecommunication Network Modeling and Planning Tool on ASP Clusters Springer Berlin / Heidelberg: Lecture Notes in Computer Science Volume 2659/2003 514-523.

[7] Clough R.W. Thoughts about the origin of the finine element method. Computers and Structures, 2001,79(22-25):2029-2030.

[8] Robert I. Levine, Diane E. Drang, Barry Edelson. AI and expert systems: a comprehensive guide to Turbo Pascal. 2nd ed. -- New York: McGraw-Hill, c1990.

[9] Heinrich C. Mayr. Database and expert systems applications. Berlin; New York: Springer-Verlag, 2001.

[10] Paul Beynon-Davies. Expert database systems: a gentle introduction London, New York: McGraw-Hill, c1991. 